\title{
Schritt für Schritt zur Blutentnahme
}

\author{
Hygienisch, rechtlich sicher und korrekt durchgeführt, ist die VENENPUNKTION für \\ Diagnose und Behandlung eine elementare Technik. Jürgen Sengebusch, Michael Herzog
}

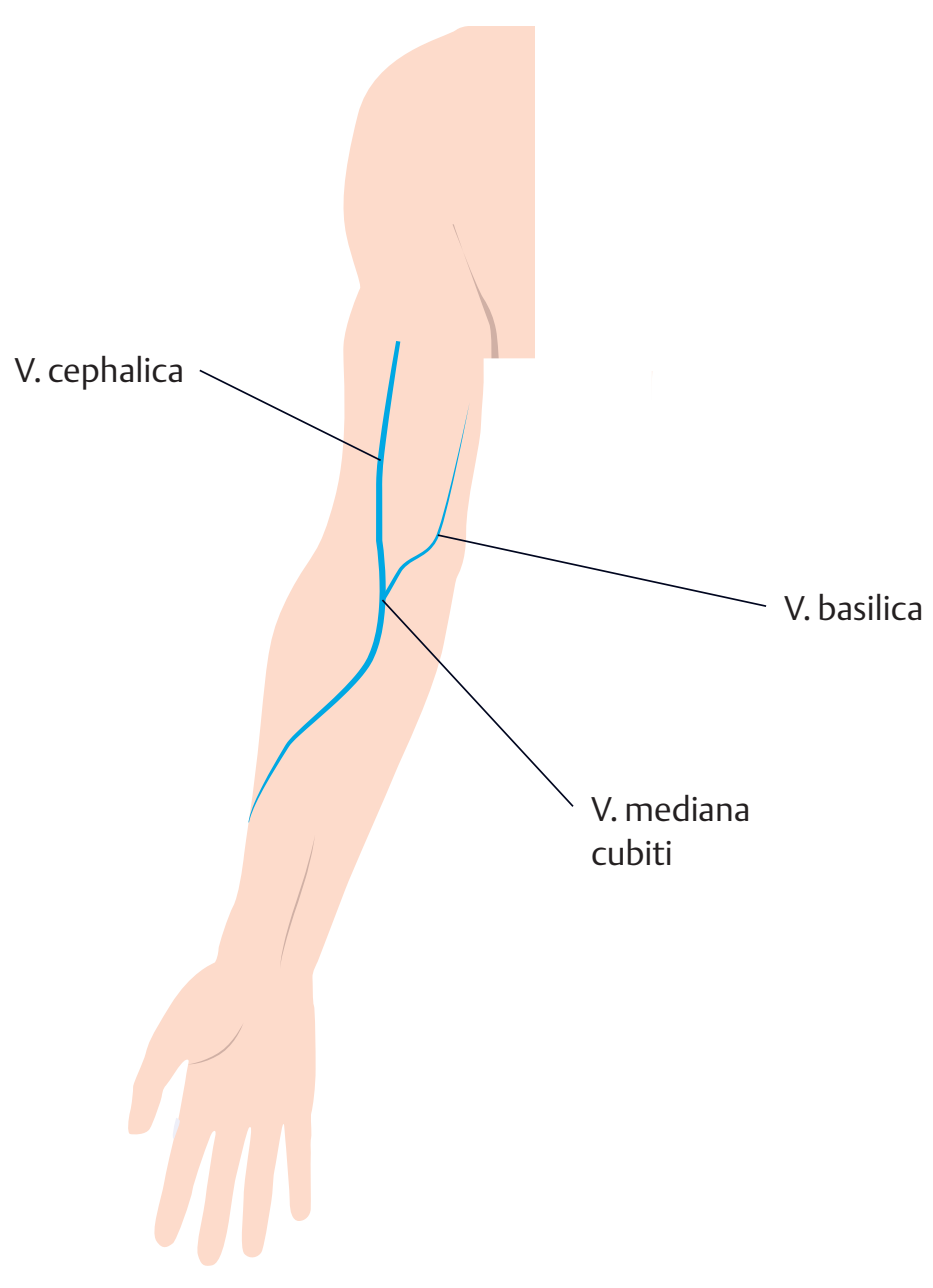

Abb. 1 Zur Venenpunktion am Arm eignen sich besonders Venen an den Ellenbeugen (V. cephalica, V. mediana cubiti), an den Unterarmen (V. basilica, V. cephalica) oder an den Handrücken. Quelle: ICare Pflege. 4. Nachdruck. Stuttgart: Thieme; 2015

\section{KURZ GEFASST}

1 Um eine sichere Diagnose zu stellen, ist eine Blutentnahme oft unerlässlich. Dafür muss in der Regel eine Vene, zum Beispiel V. cephalica, punktiert werden.

2 Vor der Venenpunktion ist es wichtig, die rechtlichen Vorschriften wie die Aufklärung des Patienten zu erfüllen sowie sorgfältig die benötigten Materialien vorzubereiten.

3 Begleitend zu einer Venenpunktion (zur Blutentnahme oder Injektion/Infusion) ist eine ordnungsgemäße Dokumentation zu leisten.

Aus der medizinischen Diagnostik ist sie kaum wegzudenken: die Blutanalyse. Sie bietet in vielen Fällen die einzige Möglichkeit für einen sicheren Befund. Eine einwandfreie Durchführung ist hierfür eine grundsätzliche Voraussetzung. In der sich anschließenden Behandlung spielen wiederum Injektions- und Infusionstherapien in vielen Praxen eine wichtige Rolle.

\section{Rechtliche Aspekte}

Grundsätzlich sollten Sie jedem Patienten bei allen Maßnahmen erläutern, was Sie tun und warum Sie es tun. Bei der Blutentnahme und Injektionen/Infusionen gilt dies in besonderem Maße: Die notwendige Venenpunktion ist ein mit Risiken verbundener Eingriff in die körperliche Unversehrtheit des Patienten. Es handelt sich - rein rechtlich betrachtet - also um eine Körperverletzung. Dem muss der Patient nach einer sorgfältigen Anamnese und Aufklärung zustimmen. Es werden zudem besondere Anforderungen an Ihr sorgfältiges hygienisches Arbeiten gestellt.

Die Aufklärung des Patienten umfasst unter anderem folgende Punkte:

- Damit der Patient der Maßnahme sicher zustimmen kann, müssen Sie ihn vorab sorgfältig über die Notwendigkeit und das Vorgehen bei der Blutentnahme und die dafür notwendige Venenpunktion aufklären. 
- Sie müssen ihm die damit verbundenen Risiken benennen.

- Sie müssen den Patienten über Alternativen unterrichten und auch über die Folgen, die es haben kann, wenn er die vorgeschlagene Maßnahme verweigert.

- Sie müssen dokumentieren, dass Sie ihn aufgeklärt haben und er der Maßnahme zugestimmt oder diese abgelehnt hat. Dasselbe gilt für die therapeutischen Maßnahmen, die mit Punktionen einhergehen.

Beobachten Sie während der Venenpunktion und Blutentnahme besonders aufmerksam, ob der Patient unerwünschte vegetative Reaktionen wie Blässe, Unruhe oder Schweißbildung zeigt. Fragen Sie ihn, wenn Sie eine Komplikation vermuten, nach seinem Befinden.

Merke: Sie dürfen kein Blut entnehmen, wenn Sie damit eine Erkrankung nachweisen möchten, die Sie als Heilpraktiker nicht behandeln dürfen - also alle im IfSG genannten Erkrankungen.

\section{Besondere Anforderungen}

Um die Sorgfaltspflicht bei der venösen Punktion zu wahren, müssen Sie

- bei der Auswahl des zu punktierenden Gefäßes über die entsprechenden anatomischen Kenntnisse verfügen.

- ausreichende Kenntnis der richtigen Venenpunktionstechniken haben.

- Vorgaben und Empfehlungen zur Hygiene der KRINKO, der TRBA 250 und gegebenenfalls weitere gesetzliche Verordnungen kennen und einhalten. Dazu müssen Sie die notwendigen Rahmenbedingungen und Materialien bereitstellen können.

- im Fall von Injektionen und Infusionen das Medizinproduktegesetz, das Arzneimittelgesetz und gegebenenfalls Anzeigepflichten gegenüber der Bezirksregierung oder des zuständigen Gesundheitsamts beachten.

- Sorge tragen, dass keine weiteren Personen (zum Beispiel andere Patienten, Mitarbeiter) gefährdet werden, beispielsweise dadurch, dass sie mit kontaminiertem Blut oder spitzen Utensilien in Berührung kommen.

- wissen, zu welchen Komplikationen es kommen kann und wie Sie sich dann richtig zu verhalten haben.

- Ihrem Patienten gegebenenfalls zuvor mitteilen, dass er zur Blutentnahme nüchtern sein muss und/oder davor bestimmte Medikamente nicht einnehmen darf.

\section{Mögliche Komplikationen}

Bei einer Venenpunktion sollten Sie mögliche Komplikation kennen und praktisch berücksichtigen. Zum Beispiel kann es passieren, dass Sie bei der Punktion die Vene durchstoßen, was zu Hämatomen führen und Schmerzen auslösen kann. Außerdem können durch falsche Punktion Nerven verletzt werden. Es besteht zudem die Gefahr, statt einer Vene eine Arterie zu punktieren. Bei Injektionen kann dies zu schmerzhaften Gefäßspasmen führen und ist unter Umständen eine Indikation, die Rettungsleitstelle hinzuziehen. Eine versehentliche intraarterielle Injektion ist jedoch höchst unwahrscheinlich, weil die Fehlpunktion bei sorgfältiger Handhabe bereits vor Beginn der Injektion deutlich wird. Darüber hinaus können vor allem bei unsachgemäßer Durchführung Keime in den Kreislauf des Patienten gelangen, die zu Infektionen führen können.

\section{Geeignete Venen für die Punktion}

Für die intravenöse Punktion wählen Sie in der Regel die Venen an den Ellenbeugen (V. cephalica, V. mediana cubiti), an den Unterarmen (V. basilica, V. cephalica) oder an den Handrücken (siehe Abb. 1). Die Venen der Fußrücken werden nur in Ausnahmen punktiert. Um die Venenfüllung zu verbessern, können Sie den Unterarm des Patienten unmittelbar vor der Punktion von distal nach proximal ausstreichen oder leicht mit Ihrem Handteller beklopfen.

Möchten Sie eine Blutentnahme vornehmen, so vermeiden Sie es, die Venendarstellung zu verbessern, indem der Patient mehrmals hintereinander die Hände zu Fäusten schließt. Die Muskelkontraktion kann dazu führen, dass die Magnesium- und KaliumSerumwerte verfälscht werden. Für eine Punktion und Injektion spielt dies keine Rolle.

Achten Sie auch darauf, insgesamt nicht länger als $1 \mathrm{~min} z u$ stauen. Eine zu lange Stauung kann zur Verfälschung von Blutwerten (zum Beispiel Kalium, LDH) und zu einer Hämolyse führen.

\section{Schritte zur Blutentnahme}

Für die Venenpunktion und die anschließende Blutentnahme bereiten Sie ein Tablett mit allen benötigten Utensilien vor. Tragen Sie hierbei Handschuhe. Bevor Sie das Spritzentablett zusammenstellen, desinfizieren Sie mit einem dafür zugelassenen Flächendesinfektionsmittel die Arbeitsfläche, auf der Sie die benötigten Utensilien gegebenenfalls ablegen, sowie das Tablett. Beachten Sie die vom Hersteller angegebene Einwirkzeit des Desinfektionsmittels.

Stellen Sie auf dem Tablett folgende Materialien bereit (siehe Abb. 2):

- passende latex- und puderfreie Einmalhandschuhe

- die für die gewünschten Laboruntersuchungen notwendigen Blutentnahmeröhrchen

- eine Blutentnahmekanüle oder einen Butterfly mit passendem Adapter

- ein VAH gelistetes Haut- und Händedesinfektionsmittel

- einen Stauschlauch

- sterilisierte Tupfer oder sterile (Einweg-)Alkoholtupfer

- Materialien für einen Kompressionsverband

- einen Spritzenabwurfbehälter und eine Nierenschale zur Ablage für Verpackungen und gebrauchte Materialien

- ein Lagerungskissen

- Halten Sie die Unterlagen für die Dokumentation bereit und vergewissern Sie sich, dass Sie im Anschluss an die Maßnahme alle Materialien umgehend sachgerecht entsorgen beziehungsweise säubern können. 


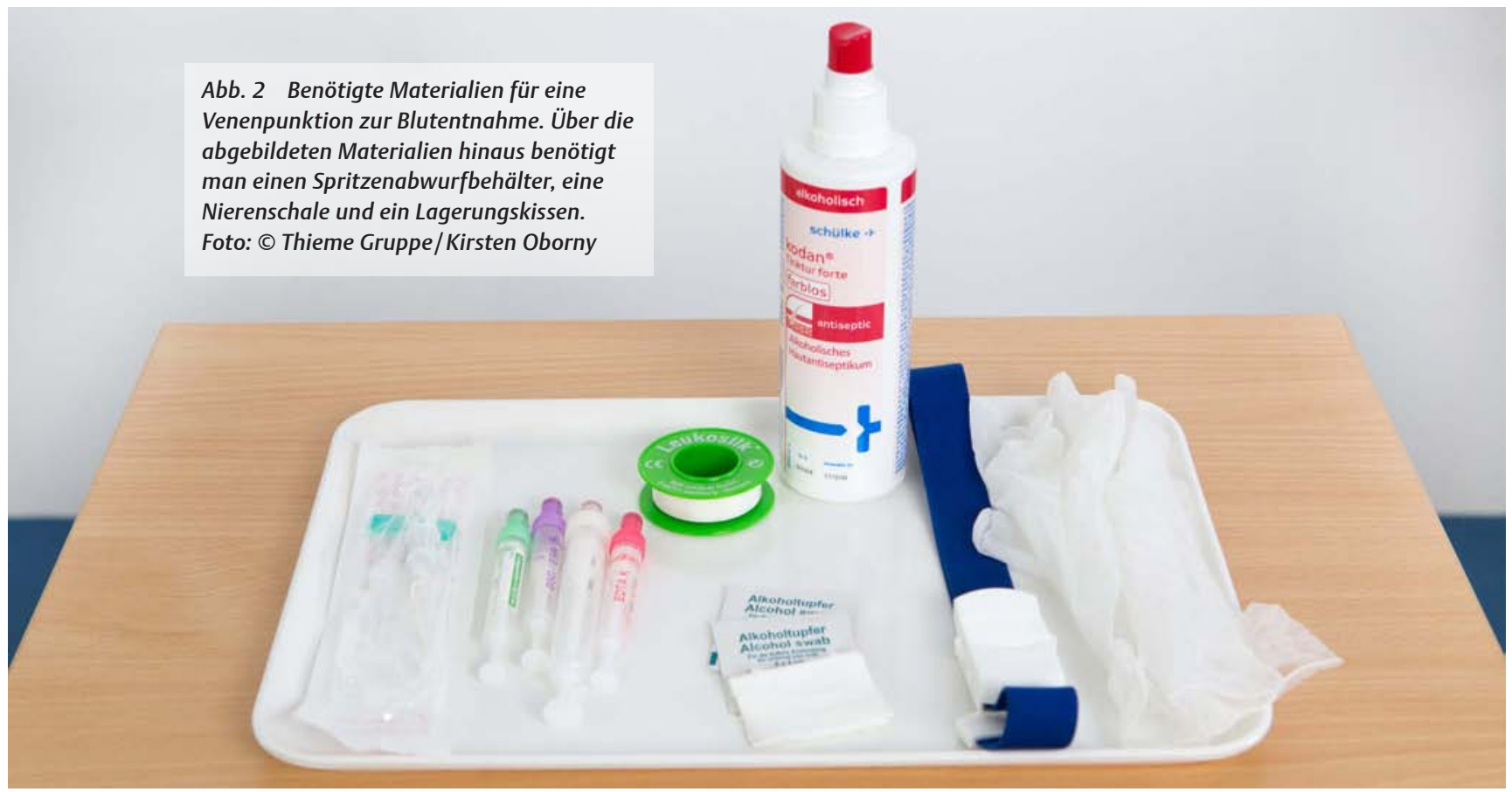

Merke: Nehmen Sie eine hygienische Händedesinfektion vor, bevor Sie steriles Material auspacken.

Öffnen Sie, wenn alles vorbereitet ist, die Verpackung der Blutentnahmekanüle. Öffnen Sie Verpackungen grundsätzlich an der dafür vorgesehenen Stelle und in der vorgesehenen Art, drücken Sie sie also niemals durch den Blister.

Entnehmen Sie die Kanüle der Packung und verbinden Sie sie über den Adapter mit dem Probeentnahmesystem, dem Blutentnahmeröhrchen. Belassen Sie die Schutzkappe in jedem Fall auf der Nadel.

\section{Lokalisation der Punktionsstelle}

Inspizieren Sie zunächst die Arme des Patienten hinsichtlich möglicher Kontraindikationen für eine Venenpunktion. Dazu zählen ein Lymphödem, ein Shunt für die Dialyse, Lähmungen, Spasmen sowie sichtbare Hautveränderungen (Entzündungen, Ödeme, Ekzeme etc.).

Platzieren Sie dann unter den für die Punktion gewählten Arm ein Lagerungs- oder Venenkissen.

Legen Sie am Oberarm des Punktionsarms einen Stauschlauch an. Achten Sie darauf, dass die Zugvorrichtung und der Verschlussmechanismus am Stauschlauch möglichst nach lateral zeigen. Das erleichtert Ihnen die Handhabung und ist für den Patienten angenehmer.

Stauen Sie das Blut, indem Sie die Zugvorrichtung anziehen und dadurch die Venen komprimieren. Achten Sie darauf, keine Hautfalten einzuklemmen. Legen Sie dazu einen Finger zwischen den Stauschlauch und den Arm des Patienten, während Sie den Stauschlauch anziehen.

Fühlen Sie dann den Radialispuls: Ist er tastbar, haben Sie die Venen gestaut. Ist er nicht tastbar, ist die Kompression zu stark und Sie haben zusätzlich die Arterie komprimiert. Lockern Sie den Stauschlauch in diesem Fall, bis der Puls wieder tastbar ist.
Inspizieren Sie nun den Arm des Patienten. Achten Sie auf gut sichtbare Venen am Unterarm und am Handrücken (siehe Abb. 1).

Die Venen an Arm und Hand zeichnen sich durch die Stauung deutlich unter der Haut ab. Bestimmen Sie durch Sichtbefund und Palpation die am stärksten gefüllte Vene. Sie ist für eine Punktion am besten geeignet. Im Zweifelsfall ist es wichtiger, eine Vene gut tasten zu können. Dies ist zuverlässiger als der Sichtbefund. Merken Sie sich die Punktionsstelle und lockern Sie den Stauschlauch.

Führen Sie dann die hygienische Händedesinfektion durch. Desinfizieren Sie die Haut über der Punktionsstelle großflächig. Empfohlen wird dazu der Gebrauch von sterilen Einmal-Alkoholtupfern. Achten Sie darauf, die Haut nach der Desinfektion nicht mehr zu berühren. Die Haut darf auch nicht durch Berührung mit dem Stauschlauch oder einem Kleidungsstück kontaminiert werden. Ansonsten müssten Sie die Hautdesinfektion wiederholen.

\section{Durchführung der Blutentnahme}

Bereiten Sie die Blutentnahme und die damit zusammenhängende venöse Punktion vor. Wenn Sie mehrere Blutentnahmeröhrchen befüllen möchten, legen Sie alle griffbereit.

Ziehen Sie Einmalhandschuhe an und legen sie den Stauschlauch wieder enger an.

Nehmen Sie das vorbereitete Blutentnahmeröhrchen mit der verbundenen Kanüle in die Hand, mit der Sie punktieren möchten. Entfernen die Schutzkappe der Kanüle mit der anderen Hand. Punktieren Sie die Vene zügig in Richtung des Venenverlaufs. Stechen Sie dabei mit der Kanüle mit nach oben zeigendem Anschliff in einem Winkel von ca. $30^{\circ}$ in die Haut und schieben Sie die Kanüle vorsichtig in die Vene. Ziehen Sie den Kolben des Blutentnahmeröhrchens leicht zurück. Aspirieren Sie dabei dunkles Blut, ist das ein Zeichen dafür, dass die Kanüle richtig in der Vene liegt. Dann können Sie die gewünschte Blutmenge langsam aspirieren. Durch zu schnelles Aspirieren können Erythrozyten platzen. 
Für den Wechsel des Blutentnahmeröhrchens halten Sie mit einer Hand den Adapter am Ansatzstück fest. Drehen Sie das Röhrchen gegen den Uhrzeigersinn und lösen Sie es damit aus dem Ansatzstück heraus. Setzen Sie das nachfolgende Blutentnahmeröhrchen auf und verbinden Sie es mit dem Ansatzstück, indem Sie es im Uhrzeigersinn eindrehen. Öffnen Sie den Stauschlauch erst, wenn Sie mit der Blutentnahme fertig sind.

Legen Sie zum Entfernen der Butterfly-Kanüle eine Kompresse auf die Punktionsstelle und ziehen Sie die Kanüle rasch aus der Vene. Legen Sie die Butterfly-Kanüle in eine dafür bereitgestellte Nierenschale. Drücken Sie einen Tupfer kräftig für ca. 2 min auf die Punktionsstelle und stoppen Sie so die Blutung. Wenn die Blutung steht, kleben Sie einen Kompressionsverband auf die Punktionsstelle. Entsorgen Sie die Kanüle sachgemäß in einem Kanülenabwurfbehälter. Entsorgen Sie gebrauchte Verpackungen und Materialien im Praxismüll.

Merke: Stecken Sie die Schutzkappe nicht wieder auf die Kanüle, Sie könnten sich dabei verletzen! Recapping ist verboten!

Zu guter Letzt: Denken Sie daran, alle Vorgänge ordnungsgemäß zu dokumentieren.

미 Dieser Artikel ist online zu finden:

http://dx.doi.org/10.1055/a-1098-6614

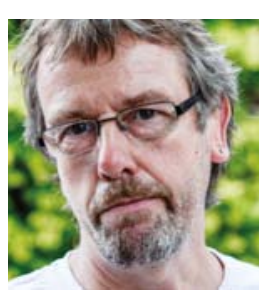

\section{HP JÜRGEN SENGEBUSCH}

Jürgen Sengebusch ist Heilpraktiker. Als Diplom-Pädagoge blickt er u.a. auf langjährige gesundheitspädagogische Arbeit in unterschiedlichen bundesweiten Einrichtungen und Verbänden zurück. Er ist Autor u.a. einiger Bücher und vieler DHZ-Artikel im Haug-Verlag. Als Dozent arbeitet er seit ca. 20 Jahren an der Hufeland-Schule Senden und betreibt zudem eine umfangreiche Online-Lernplattform zur Vorbereitung auf die HeilpraktikerÜberprüfung.

E-Mail: info@artuweise.de; info@heilpraktikerlernzentrum.de

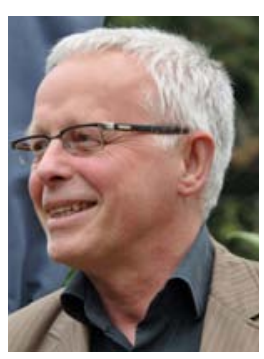

\section{HP MICHAEL HERZOG}

Michael Herzog ist Diplom-Theologe und Heilpraktiker. Seit 1988 ist er in eigener Praxis u. a. mit den Schwerpunkten Augendiagnose, klassische Naturheilverfahren, und Colon-Hydro-Therapie tätig. Er ist Autor u.a. einiger Bücher und vieler DHZ-Artikel im Haug-Verlag. Seit 1991 Referent in der Heilpraktikerausbildung. Er ist Gründer und Leiter der Hufeland-Schule Senden.

E-Mail: michael.herzog@hufelandschule.de 